\title{
Human Neutrophil Heterogeneity Identified Using Flow Microfluorometry to Monitor Membrane Potential
}

\author{
Bruce Seligmann, Thomas M. Chused, and John I. Gallin, Bacterial Diseases \\ Section, Laboratory of Clinical Investigation, and the Laboratory of Microbial \\ Immunity, National Institute of Allergy and Infectious Diseases, National \\ Institutes of Health, Bethesda, Maryland 20205
}

A B S T RACT Previous studies of neutrophil nitroblue tetrazolium dye reduction in response to endotoxin and rosetting of IgG-coated erythryocytes have suggested functional heterogeneity of peripheral blood neutrophils. In the following study we utilized flow microfluorometry and the membrane potentialsensitive fluorescent dye 3-3'-dipentyloxacarbocyanine to assess the heterogeneity of neutrophils upon activation by a variety of stimuli. Unstimulated neutrophils from normal subjects exhibited a unimodal distribution of fluorescence, suggesting that all the cells possessed the same resting membrane potential. As neutrophils aged $(>5 \mathrm{~h}$ ), some cells lost fluorescence producing a bimodal distribution. In studies with fresh cells, the secretagogue phorbol myristate acetate $(20$ $\mathrm{ng} / \mathrm{ml}$ ) stimulated a uniform loss of fluorescence (apparent depolarization). The chemoattractant $N$-formylmethionyl-leucyl-phenylalanine (f-Met-Leu-Phe) $(0.1 \mu \mathrm{M})$ caused the neutrophils to assume and maintain (for $>30 \mathrm{~min}$ ) a bimodal fluorescence distribution in which $65 \pm 5 \%$ of the neutrophils first decreased and then increased fluorescence (apparent depolarization/ partial repolarization), and $35 \pm 5 \%$ of the cells exhibited either an increase in fluorescence (apparent hyperpolarization) or no change. Treatment of neutrophils with cytochalasin $B$ before stimulation caused the cells to respond homogeneously to f-Met-Leu-Phe. Additional studies using neutrophils from patients with chronic granulomatous disease, which exhibit abnormal membrane potential responses, indicated that this defect affected all such neutrophils uniformly. These observations demonstrate the need to investigate the physiological significance of the heterogeneity of neutrophil function and indicate that the f-Met-LeuPhe-induced changes in membrane potential observed

Address reprint requests to Dr. Bruce Seligmann, National Institutes of Health, Bethesda, Md. 20205.

Received for publication 4 May 1981 and in revised form 6 July 1981. in bulk population cell studies are the summation of two different responses.

\section{INTRODUCTION}

Polymorphonuclear leukocytes (neutrophils) ${ }^{1}$ obtained from peripheral blood exhibit a variety of functional responses to activating agents. These include adherence to surfaces, cell aggregation, chemotaxis, secretion of granule contents, phagocytosis, and generation of reactive molecules such as superoxide anion $\left(\mathrm{O}_{2}^{\bar{\prime}}\right)$ and peroxide $(1,2)$. Previous work from this laboratory and others has indicated that there is heterogeneity of human neutrophils. Klempner and Gallin (3) reported that $20 \%$ of neutrophils had poor adherence and chemotaxis and that this subpopulation had decreased Fc receptor expression as detected by the failure to form rosettes with opsonized erythrocytes. Broxmeyer et al. (4) have suggested that neutrophils with readily expressed Fc receptors may be important in the regulation of myelopoiesis. Furthermore, in response to endotoxin, a significant number of cells do not reduce nitroblue tetrazolium (NBT), a property associated with production of $\mathrm{O}_{2}^{\bar{i}}$. In contrast, all cells reduce NBT when treated with the secretagogue phorbol myristate acetate (PMA) (5). These observations all support the thesis that human neutrophils are heterogeneous.

In this report, we describe another aspect of human neutrophil heterogeneity. We utilized flow microfluorometry (FMF) and a fluorescent probe, 3-3'dipentyloxacarbocyanine [di-O- $\left.\mathrm{C}_{5}(3)\right](6)$. The intensity of fluorescence of this dye reflects the cell membrane potential and, unlike a related probe (3-3'-dipropylthiodicarbocyanine), di-O- $\mathrm{C}_{5}(3)$ fluorescence is not af-

\footnotetext{
${ }^{1}$ Abbreviations used in this paper: CGD, chronic granulomatous disease; di-O- $\mathrm{C}_{5}(3), 3-3$ '-dipentyloxacarbocyanine; f-Met-Leu-Phe, N-formyl-methionyl-leucyl-phenylalanine; FMF, flow microfluorometry; NBT, nitroblue tetrazolium; neutrophils, polymorphonuclear leukocytes; $\mathrm{O}_{2}^{\bar{i}}$, superoxide anion; PMA, phorbol myristate acetate.
} 
fected by reactive products of neutrophil oxidative metabolism such as $\mathrm{O}_{2}^{\bar{i}}$ or hydrogen peroxide (7). This and similar probes have only recently been used with FMF to monitor the membrane potential of individual cells (6-9). We report here that the apparent membrane potential responses of individual neutrophils to PMA is homogeneous while that to $\mathrm{N}$-formyl-methionylleucyl-phenylalanine (f-Met-Leu-Phe) is heterogeneous. Such heterogeneity must be considered when evaluating data from experiments with bulk cell populations.

\section{METHODS}

Assays were carried out in phosphate-buffered Hanks' solution as described previously $(10-12)$. The croton oil derivative PMA (Consolidated Midland Corp., Brewster, N. Y.), f-MetLeu-Phe (Sigma Chemical Co., St. Louis, Mo.) and cytochala$\sin$ B (Aldrich Chemical Co., Milwaukee, Wis.) were dissolved in dimethylsulfoxide (Fisher Scientific Co., Pittsburgh, Pa.) to make stock solutions such that the final concentration of dimethylsulfoxide in the reaction mixture was $0.1 \%$. This amount of dimethylsulfoxide had no effect on secretion, $\mathrm{O}_{2}^{-}$ generation, cell viability as measured by trypan blue exclusion and lactate dehydrogenase release, or the fluorescence responses.

Additional reagents included Hypaque, Ficoll, and T250 dextran (Pharmacia, Uppsala, Sweden). Dr. Alan Waggoner, Amherst College, Amherst, Mass., kindly provided di-O- $\mathrm{C}_{5}(3)$, which was dissolved in ethanol to prepare stock solutions from which at least 1,000-fold dilutions were made.

Leukocyte isolation. Peripheral blood neutrophils from normal human subjects were prepared by Ficoll-Hypaque gradient centrifugation followed by dextran sedimentation (13). This technique routinely resulted in cell populations containing $>95 \%$ neutrophils. All experiments were completed within $5 \mathrm{~h}$ of obtaining the cells ( $2 \mathrm{~h}$ after purification).

Flow microfluorometric assay of individual cell membrane potential. FMF was performed with a FACS II (Becton, Dickinson \& Co., Rutherford, N. J.) using an argon ion laser, emitting $200 \mathrm{~mW}$ at $488 \mathrm{~nm}$. Narrow angle forward light scatter was used for live/dead discrimination and as a measure of cell size. The data were analyzed by a PDP 11/34 (Digital Equipment Corp., Marlboro, Mass.). Before assay the neutrophils were kept on ice in the absence of $\mathrm{Ca}^{+2}$ and $\mathrm{Mg}^{+2}$. These conditions reduce neutrophil self-aggregation as well as the spontaneous secretion of granule contents and generation of $\mathrm{O}_{2}^{\bar{\gamma}} .15 \mathrm{~min}$ before assay neutrophils were suspended at $10^{6} / \mathrm{ml}$ with stirring at $37^{\circ} \mathrm{C}$ in phosphate-buffered Hanks' solution containing physiologic amounts of $\mathrm{NaCl}(129 \mathrm{mM}), \mathrm{KCl}(4.2$ $\mathrm{mM}), \mathrm{Ca}^{+2}(1.6 \mathrm{mM}), \mathrm{Mg}^{+2}(0.3 \mathrm{mM})$, glucose $(5 \mathrm{mM})$, phosphate $(1.5 \mathrm{mM})$, and sulfate $(0.4 \mathrm{mM})$ to allow the cells to reach thermal and ionic equilibrium. The fluorescent probe di-O- $\mathrm{C}_{5}(3)$ was then added $(10 \mathrm{nM})$ and the neutrophils allowed to equilibrate, which required $\sim 5 \mathrm{~min}$. Three activating agents were used: the secretagogue PMA $(20 \mathrm{ng} / \mathrm{ml})$, the chemoattractant f-Met-Leu-Phe $(0.1 \mu \mathrm{M})$, and f-Met-LeuPhe $(0.1 \mu \mathrm{M})$ plus cytochalasin $\mathrm{B}(5 \mu \mathrm{g} / \mathrm{ml})$. The results are presented as the distribution of fluorescence from neutrophils equilibrated with di-O- $\mathrm{C}_{5}(3)$ before ( 0 time) and at various times after addition of stimuli. The data are presented as fluorescence distributions of 20,000 or more cells. The percentage of cells per channel is plotted on the vertical axis against fluorescence, divided into 1,000 linear channels, on the horizontal axis.

\section{RESULTS}

Normal neutrophils. In 19 of 21 experiments, neutrophils equilibrated with di-O- $\mathrm{C}_{5}(3)$ and assayed within $2 \mathrm{~h}$ of purification exhibited a unimodal distribution of fluorescence (Fig. 1A), suggesting that all the cells had the same resting membrane potential. In 2 of the 21 experiments a bimodal distribution was observed (Fig. 1B). Cells from each peak of fluorescence for these two subjects were collected and both contained $>95 \%$ mature neutrophils. Because previous studies using neutrophils in cell suspension indicated that "aging" of neutrophils after purification caused an apparent membrane depolarization $(10,12)$, similar studies were conducted using FMF to determine if the heterogeneity noted in these two cell preparations could be induced from an initial unimodal preparation by letting the cells incubate in vitro. Normal neutrophils with an initial unimodal distribution were incubated at $37^{\circ} \mathrm{C}$ in the presence of $\mathrm{Ca}^{+2}$ and $\mathrm{Mg}^{+2}$ and assayed periodically beginning at $3 \mathrm{~h}$. The results are shown in Fig. 2. Cells assayed immediately after a 3-h purification or after a 1.5 -h incubation at $37^{\circ} \mathrm{C}(4.5-\mathrm{h}$ point, Fig. 2) displayed a unimodal fluorescence distri-



Figure 1 Distribution of equilibrium fluorescence of neutrophils before exposure to activating stimuli. (A) Unimodal distribution observed in 19 of 21 experiments. (B) Bimodal distribution seen in 2 of 21 experiments. 
bution that subsequently changed to a distinct bimodal distribution after an additional 45-min incubation at $37^{\circ} \mathrm{C}(5.25$-h point, Fig. 2). Viability of unseparated cells ( $>95 \%$ by trypan exclusion) was unchanged during this experiment. The light scatter of cells from both peaks was the same, indicating that the acquired heterogeneity of "aging" was not due to cell aggregation or clumping. Thus the bimodal distribution seen in the two experiments from cells assayed $<1 \mathrm{~h}$ after purification may reflect rapid aging of the cells. Whether this reflects an artifact induced by neutrophil purification and/or storage is not known. The aging process may actually reflect recovery of cells from the purification procedure. To avoid this complication, we restricted subsequent experiments to cells exhibiting an initial unimodal fluorescence distribution.

Effect of activating stimuli. Activation of neutrophils with the secretagogue PMA $(20 \mathrm{ng} / \mathrm{ml})$ caused, within $5 \mathrm{~min}$, a uniform decrease in fluorescence (Fig. 3A) which persisted for $>30 \mathrm{~min}$. A similar homogeneous response was obtained using a second secretagogue, the calcium ionophore $\mathrm{A} 23187(0.5 \mu \mathrm{M}$; results not shown). In contrast to the uniform response to PMA, exposure to the chemoattractant f-Met-Leu-Phe (0.1 $\mu \mathrm{M})$ induced the appearance of two peaks of fluorescent cells (Fig. 3B). Approximately $35 \%$ of cells exhibited no change or a small increase in fluorescence (apparent hyperpolarization) relative to the initial resting fluorescence level (channel number 680). The remainder of neutrophils $(65 \%)$ exhibited a large decrease in fluorescence followed by a more gradual increase (apparent depolarization followed by a slow partial repolarization). A conclusive demonstration that these two different responses seen with FMF represent actual changes in membrane potential awaits their detailed ionic characterization. Therefore we refer to the responses as "apparent" changes in membrane potential. 10 min after stimulation the bimodal fluorescence distribution stabilized with peaks at channels 400 and 800 (Fig. 3B, $10 \mathrm{~min}$ ). In fact, these neutrophil populations remained distinct for up to $30 \mathrm{~min}$. As demonstrated in Table I, in which the results from eight different donors are shown, the percentage of cells in each population of neutrophils varied from donor to donor, but the difference between the number of responding and nonresponding cells was significant.

It is evident from Table I that the ratio of the number of cells responding to f-Met-Leu-Phe with an apparent hyperpolarization to the number of cells responding with an apparent depolarization is variable from experiment to experiment. To determine whether this variability was a consequence of day-to-day variability (due to handling of cells and purification differences) or possibly related to person-to-person variability, we obtained data using one donor (B.S.) on $5 \mathrm{~d}$ and performed an analysis of variance using the com-

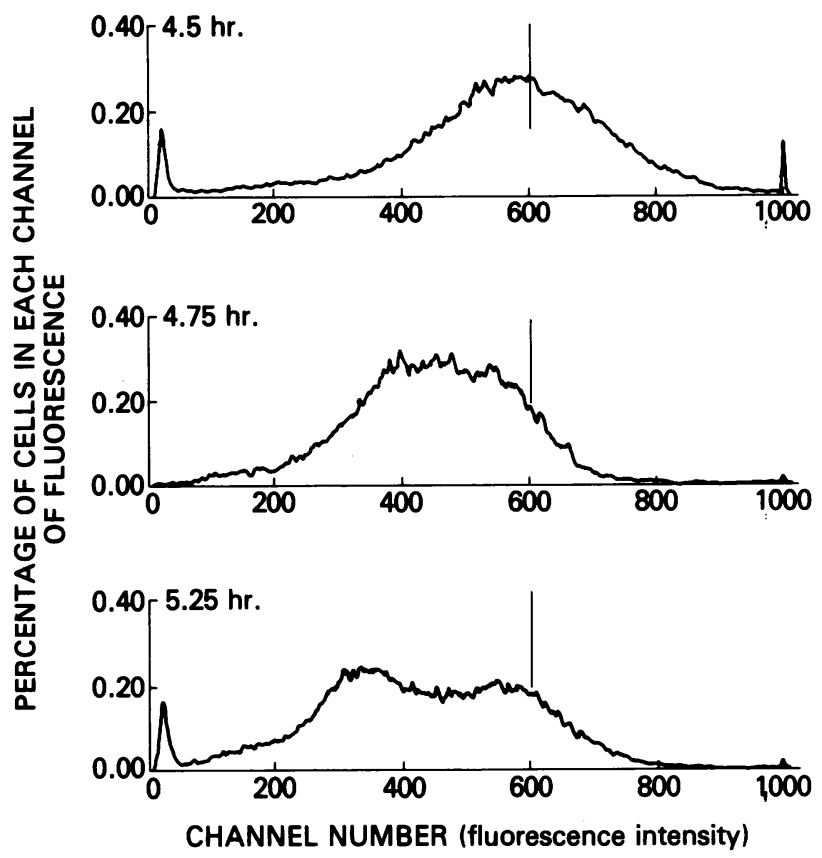

Figure 2 Effect of neutrophil age on the equilibrium fluorescence distribution of unstimulated neutrophils incubated in the presence of $\mathrm{Ca}^{+2}$ and $\mathrm{Mg}^{+2}$ at $37^{\circ} \mathrm{C}$ beginning $3 \mathrm{~h}$ after drawing the blood sample. The cells were assayed periodically at the times indicated after blood drawing. Note two peaks of fluorescence (channels 580 and 340 ), $5.25 \mathrm{~h}$ after drawing blood. The small peak at " 0 " fluorescence in these figures was composed of erythrocytes and dead cells and was unaffected by changing dye concentration.

ponents-of-variance Model II (14). In this model the total variance of an individual observation is expressed as a sum of its component variabilities, in this case dayto-day variability $\left(\sigma^{2}\right)$ and person-to-person variability $\left(\sigma_{p}^{2}\right)$. It was found that the variability from person to person expressed as the standard deviation $\left(\hat{\sigma}_{\mathrm{p}}=15.0\right)$ was greater than day-to-day variability $\left(\hat{\sigma}_{\mathrm{D}}=11.0\right)$. This suggests that person-to-person differences in response ratios exist and are being detected.

The two populations of neutrophils evident after f-Met-Leu-Phe stimulation were sorted and collected 10 min after stimulation and both contained $>93 \%$ mature, viable neutrophils. Immature neutrophils were not distributed preferentially between the two groups of cells. Thus the appearance of two cell populations could not be attributed to different responses from contaminating cells. In addition, the light scatter distribution of neutrophils from both populations was identical.

In studies designed to relate the heterogeneous f-Met-Leu-Phe di-O- $\mathrm{C}_{5}(3)$ response to cell function, the effect of cytochalasin B was investigated. Cytochalasin B treatment of neutrophils not only causes secretion upon subsequent stimulation with f-Met-Leu-Phe but also enhances the production of $\mathrm{O}_{2}^{-}$by f-Met-Leu-Phe 


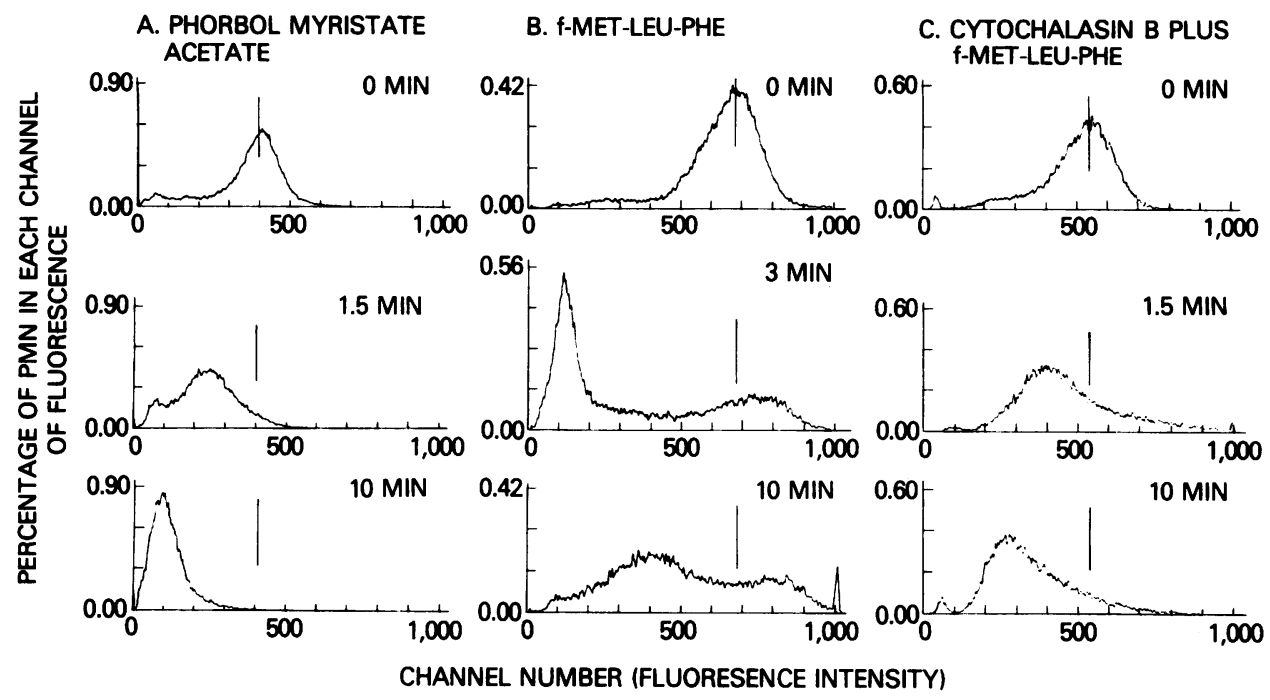

FIGURE 3 Effect of various stimuli on neutrophil fluorescence. (A) (0 time) shows the pattern of fluorescence of neutrophils before stimulation. The effect of the secretagogue PMA $(20 \mathrm{ng} / \mathrm{ml})$ on neutrophil fluorescence is shown at 1.5 and $10 \mathrm{~min}$ after its addition. The distribution observed at $10 \mathrm{~min}$ remained stable for up to $30 \mathrm{~min}$ following stimulation. (B) Effect of the chemoattractant f-Met-Leu-Phe $(0.1 \mu \mathrm{M})$ on neutrophil di-O- $\mathrm{C}_{5}(3)$ fluorescence. The distribution of fluorescence from individual neutrophils is shown before $(0$ time $)$ and 3 and 10 min following addition of f-Met-Leu-Phe. (C) Effect of cytochalasin B on neutrophil response to f-Met-Leu-Phe. Neutrophils were treated with $5 \mu \mathrm{g} / \mathrm{ml}$ cytochalasin B for $5 \mathrm{~min}$ before stimulation. Fluorescence distribution patterns were subsequently obtained, immediately before addition of f-Met-Leu-Phe $(0.1 \mu \mathrm{M}, 0$ time) and at 1.5 and $10 \mathrm{~min}$ after its addition.

$(1,2)$. Treatment of neutrophils for 5 min with cytochalasin B $(5 \mu \mathrm{g} / \mathrm{ml})$ caused the cells to respond homogeneously when subsequently stimulated with f-MetLeu-Phe, with all the neutrophils exhibiting a uniform decrease in fluorescence (Fig. 3C). If cytochalasin B

TABLE I

Variability among Different Neutrophil Preparations in di-O-C $\mathrm{C}_{5}(3)$ Responses to f-Met-Leu-Phe Assessed by FMF

\begin{tabular}{ccc}
\hline & \multicolumn{2}{c}{ Percentage of responding neutrophils } \\
\cline { 2 - 3 } $\begin{array}{c}\text { Neutrophil } \\
\text { preparation }\end{array}$ & Decreased fluorescence & $\begin{array}{c}\text { Increase or no change } \\
\text { in fluorescence }\end{array}$ \\
\hline M.B. & 75 & 25 \\
N.M. & 55 & 45 \\
S.B. & 60 & 40 \\
F.M. & 50 & 50 \\
I.D. & 91 & 9 \\
D.V. & 70 & 30 \\
R.H. & 75 & 25 \\
B.S. & 44 & 55 \\
Mean \pm SEM & $65 \pm 5 \ddagger$ & $35 \pm 5 \ddagger$ \\
\hline
\end{tabular}

* The average distribution $\pm \mathrm{SEM}$ for all experiments is indicated at the bottom of the table.

$\ddagger P<0.001$, level of difference between percentage of cells showing decreased fluorescence vs. increased or no change in fluorescence, Student's $t$ test. was added to cells 7 min after addition of f-Met-LeuPhe, the nonresponding cells responded with a decrease in fluorescence, causing the f-Met-Leu-Pheinduced bimodal response to become unimodal. These data raise the interesting possibility that the enhanced degranulation and $\mathrm{O}_{2}^{\bar{z}}$ production induced by cytochalasin $B$ represents recruitment of responses from additional cells rather than or in addition to increasing the magnitude of the response of individual cells.

Neutrophils from patients with chronic granulomatous disease (CGD). Neutrophils from patients with CGD fail to produce $\mathrm{O}_{2}^{\overline{\overline{ }}}$ and also fail to generate normal responses to membrane potential-sensitive probes when stimulated with PMA or f-Met-Leu-Phe $(12,15)$. Studies using FMF were performed on neutrophils obtained from two patients with CGD to determine if the abnormal response represents a uniform defect of all cells or summation of heterogeneous responses (Fig. 4). Neutrophils from both patients with CGD were uniform, with all cells producing an abnormally small response to both PMA and f-Met-Leu-Phe compared with that produced by neutrophils from the normal control. The response of neutrophils from one patient is shown in Fig. 4B.

\section{DISCUSSION}

The di-O- $\mathrm{C}_{5}(3)$ fluorescence of unstimulated normal neutrophils is homogeneous and compatible with a 



CHANNEL NUMBER (FLUORESCENCE INTENSITY)

Figure 4 Effect of f-Met-Leu-Phe $(0.1 \mu \mathrm{M})$ and PMA $(20 \mathrm{ng} / \mathrm{ml})$ on fluorescence of neutrophils (A) obtained from a normal control donor and (B) obtained from a patient with CGD. Fluorescence distribution patterns were obtained immediately before stimulation ( 0 time) and 4 min after stimulation with either f-Met-Leu-Phe or PMA, as indicated for each tracing.

relatively high resting membrane potential (16). As normal neutrophil age, an increasing number of cells lose fluorescence (apparent depolarization), resulting in a bimodal distribution of fluorescence (Fig. 2). In studies using indirect molecular probes of membrane potential or bulk populations, the estimated resting membrane potential of young neutrophils has been reported previously to be about $-50 \mathrm{mV}(16)$ and during neutrophil aging a reduction of membrane potential to $\sim-25 \mathrm{mV}$ occurs. However, as shown in the current studies, these estimations of the resting membrane potential of populations of unaged and aged neutrophils may reflect the summation of at least two populations.

The results presented here indicate that neutrophils respond uniformly with decreased fluorescence (membrane potential depolarization) when activated by PMA. In contrast, neutrophils respond heterogeneously to f-Met-Leu-Phe. The majority (65\%) of cells respond with a decrease followed by a partial return to the original fluorescence level. The remainder $(35 \%)$ either 
do not respond or show an increase in fluorescence. This dimorphic response to f-Met-Leu-Phe was independent of cell age since older cells, provided their di$\mathrm{O}_{-} \mathrm{C}_{5}(3)$ fluorescence had not already decreased (depolarized), also exhibited both responses (data not shown). The heterogeneity could not be attributed to contamination of the cell preparations with other cell types or the presence of immature neutrophils. The differences also could not be attributed to dimerization of di-O- $\mathrm{C}_{5}(3)$ within some cells because the responses were independent of di-O- $\mathrm{C}_{5}(3)$ concentration (data not shown) (10). The results presented here, therefore, indicate that the previously reported apparent changes in neutrophil membrane potential caused by f-MetLeu-Phe, which have been based on studies using bulk cell suspensions $(10-12,16-19)$, represent the summation of two different responses. Ionic studies performed using whole populations of neutrophils (20) must therefore be reassessed to consider the contribution of neutrophil heterogeneity.

The heterogeneity of the f-Met-Leu-Phe di-O- $\mathrm{C}_{5}(3)$ response may reflect populations of neutrophils with different availability, affinity, or functional activity of the f-Met-Leu-Phe receptor. The ability of cytochalasin $B$ to condition neutrophils so that upon stimulation with f-Met-Leu-Phe the cell population responds homogeneously may reflect either direct effects of cytochalasin B, or indirect effects of products secreted or reactive species generated in response to cytochalasin B. The previously reported ability of cytochalasin B to amplify neutrophil di-O- $\mathrm{C}_{5}(3)$ responses to f-Met-LeuPhe (11) would therefore appear to be explained, at least in part, by mobilization of nonresponding cells rather than just amplification of individual cell responsiveness.

Whether or not the heterogeneity of neutrophil di$\mathrm{O}_{-} \mathrm{C}_{5}(3)$ fluorescence response to $\mathrm{f}-\mathrm{Met}-\mathrm{Leu}-\mathrm{Phe}$ is related to the previously published heterogeneity of neutrophil Fc receptor expression (3) could not be adequately addressed. Cells stimulated with f-Met-LeuPhe and separated by FMF tended to aggregate, reducing the reliability of the rosetting assay of $\mathrm{Fc}$ receptor expression, while cells separated by differences in rosetting with Ig-coated erythrocytes (3) had begun to "age" and exhibited diminished di-O- $\mathrm{C}_{5}(3)$ fluorescence as described in Fig. 2.

The heterogeneity of the neutrophil di-O- $\mathrm{C}_{5}(3)$ fluorescence response to f-Met-Leu-Phe may be related to heterogeneity of endotoxin-stimulated NBT reduction (5). Neutrophil NBT reduction in the presence of both endotoxin and f-Met-Leu-Phe $(1 \mu \mathrm{M})$ is heterogeneous with only $\sim 40 \%$ of cells reducing NBT. Treatment with cytochalasin $B$ resulted in NBT reduction by $>95 \%$ of cells in response to f-Met-Leu-Phe, ${ }^{2}$ similar to

\footnotetext{
${ }^{2}$ Seligmann, B., and J. I. Gallin. Unpublished observations.
}

the response seen with PMA (5). The similarity between NBT reduction in response to f-Met-Leu-Phe in the absence or presence of cytochalasin $B$ and that of the neutrophil di-O- $\mathrm{C}_{5}(3)$ fluorescence response reported in this communication suggests that the heterogeneity of NBT reduction and di-O- $\mathrm{C}_{5}(3)$ responsiveness may be related. In this regard, it is of interest that the previously reported abnormality of CGD neutrophil O-C ${ }_{5}(3)$ response to f-Met-Leu-Phe and PMA (12, 15), like the abnormality of NBT reduction in these patients, is a defect of all the neutrophils obtained from the particular CGD patients studied.

The adaptation of FMF and fluorescent probes of membrane potential $(6,9)$ provides a new method for addressing both the functional significance of normal neutrophil heterogeneity and for characterizing abnormal responses seen in neutrophils obtained from certain patients.

\section{ACKNOWLEDGMENT}

The authors thank Dr. David Alling (Office of the Scientific Director, National Institute of Allergy and Infectious Diseases, National Institutes of Health, Bethesda, Maryland) for performing the analysis of variance.

\section{REFERENCES}

1. Klebanoff, S. J., and R. A. Clark. 1978. The Neutrophil: Functional and Clinical Disorders. Elsevier Inc., NorthHolland, New York.

2. Weissmann, G. 1980. The Cell Biology of Inflammation; Handbook of Inflammation 2. Elsevier North-Holland Inc., New York.

3. Klempner, M. S., and J. I. Gallin. 1978. Separation and functional characterization of human neutrophil subpopulations. Blood. 51: 659-669.

4. Broxmeyer, H. E., P. Ralph, J. Bognachi, P. W. Kinkade, and M. Desousa. 1980. A subpopulation of human polymorphonuclear neutrophils contains an active form of lactoferrin capable of binding to human monocytes and inhibiting production of granulocyte-macrophage colony stimulatory activity. J. Immunol. 125: 903-909.

5. Repine, J. E., B. Rasmussen, and J. G. White. 1979. An improved nitroblue tetrazolium test using phorbol myristate acetate-coated coverslips. Am. J. Clin. Pathol. 71: 582-585.

6. Seligmann, B., and J. I. Gallin. 1981. Human neutrophil heterogeneity identified using the fluorescent membrane potential probe di-O- $\mathrm{C}_{5}(3)$ and flow microfluorometry. Clin. Res. 29: 522. (Abstr.)

7. Seligmann, B., and J. I. Gallin. Neutrophil activation studied using two indirect probes of membrane potential which respond by different fluorescence mechanisms. In Advances in Experimental Medicine and Biology: Biochemistry and Function of Phagocytes. P. Patriarea, and F. Rossi, editors. Plenum Publishing Corporation, New York. 141.

8. Seligmann, B., M. Garriga, W. M. Leiserson, and T. M. Chused. 1981. Membrane potential analysis of murine lymphocyte subsets by flow microfluorometry utilizing charge sensitive dyes. Fed. Proc. 40: 4567 (Abstr.).

9. Shapiro, H. M., P. J. Natale, and L. A. Kamentsby. 1979. Estimation of membrane potentials of individual 
lymphocytes by flow cytometry. Proc. Natl. Acad. Sci. U. S. A. 76: 5728-5730.

10. Seligmann, B., E. Gallin, D. Martin, W. Shain, and J. I. Gallin. 1980. Interaction of chemotactic factors with human polymorphonuclear leukocytes: studies using a membrane potential-sensitive cyanine dye. J. Membr. Biol. 52: 257-272.

11. Seligmann, B., and J. I. Gallin. 1980. Secretagogue modulation of the response of human neutrophils to chemoattractants: studies with a membrane potential sensitive cyanine dye. Mol. Immunol. 17: 191-200.

12. Seligmann, B., and J. I. Gallin. 1980. Use of lipophilic probes of membrane potential to assess human neutrophil activation: abnormality in chronic granulomatous disease. J. Clin. Invest. 66: 493-503.

13. Boyum, A. 1978. Isolation of mononuclear cells and granulocytes from human blood. Scand. J. Clin. Lab. Invest. 97(Suppl. 29): 77-89.

14. Dixon, W. S., and F. J. Clark, Jr. 1969. Introduction to Statistical Analysis. McGraw-Hill Ryerson Ltd., New York. 3rd edition. 302-308.

15. Whitin, J., C. Chapman, E. Simons, M. Chovaniec, and H. Cohen. 1980. Correlation between membrane potential changes and superoxide production in human granulocytes stimulated by phorbol myristate acetate. J. Biol. Chem. 255: 1874-1878.
16. Seligmann, B., and J. I. Gallin. 1981. Use of indirect probes of membrane potential in human neutrophils: comparison of di-O- $\mathrm{C}_{5}(3)$ and triphenylmethylphosphonium ion. In Membranes and Cytoskeleton: Role in Pathologic Processes. N. S. McNutt and S. Hoffstein, editors. Fed. Proc. 40: 211-212.

17. Jones, G. S., K. Van Dyke, and V. Castranova. 1980. Purification of human granulocytes by centrifugal elutriation and measurement of transmembrane potential. J. Cell Physiol. 104: 425-431.

18. Simchowitz, L., J. P. Atkinson, and I. Spilberg. 1980. Stimulus-specific deactivation of chemotactic factorinduced cyclic AMP response and superoxide generation by human neutrophils. J. Clin. Invest. 66: 736-747.

19. Tatham, P., P. Delves, L. Shen, and I. Roitt. 1980. Chemotactic factor-induced membrane potential changes in rabbit neutrophils monitored by the fluorescent dye 3,3'dipropylthiodicarbocyanine iodide. Biochim. Biophys. Acta. 602: 285-298.

20. Naccahe, P. H., H. J. Showell, E. L. Becker, and R. I. Sha'afi. 1979. The chemotactic factors induced movement of calcium and sodium across rabbit neutrophil membranes: effect of desensitization to cytochalasin B. J. Cell Physiol. 100: 239-250. 